\title{
Multiple stochastic volatility extension of the Libor market model and its implementation *
}

\author{
Denis Belomestny ${ }^{1}$, Stanley Mathew ${ }^{2}$, and John Schoenmakers ${ }^{1}$
}

February 19, 2009

Keywords: Libor modeling, stochastic volatility, CIR processes, calibration

AMS 2000 Subject Classification: 60G51, 62G20, 60H05, 60H10, 90A09, 91B28 JEL Classification Code: G12

\begin{abstract}
In this paper we propose an extension of the Libor market model with a high-dimensional specially structured system of square root volatility processes, and give a road map for its calibration. As such the model is well suited for Monte Carlo simulation of derivative interest rate instruments. As a key issue, we require that the local covariance structure of the market model is preserved in the stochastic volatility extension. In a case study we demonstrate that the extended Libor model allows for stable calibration to the cap-strike matrix. The calibration algorithm is FFT based, so fast and easy to implement.
\end{abstract}

\section{Introduction}

Since Brace, Gatarek, Musiela (1997), Jamshidian (1997), and Miltersen, Sandmann and Sondermann (1997), almost independently, initiated the development of the Libor market interest rate model, research has grown immensely towards improved models that fit market quotes of standard interest rate products such as cap and swaption prices for different strikes and maturities. As a matter of fact, while caps can be priced using a Black type formula and swaptions via closed form approximations with high accuracy, an important drawback of the market model is the impossibility of matching cap and swaption volatility

\footnotetext{
${ }^{1}$ Weierstrass Institute for Applied Analysis and Stochastics, Mohrenstr. 39, 10117 Berlin, Germany. \{belomest, schoenma\}@wias-berlin.de.

${ }^{2}$ Johann Wolfgang Goethe-Universität, Senckenberganlage 31, 60325 Frankfurt am Main, Germany. mathew@math.uni-frankfurt.de.

* Partial support by the Deutsche Forschungsgemeinschaft through the SFB 649 'Economic Risk' and the DFG Research Center Matheon 'Mathematics for Key Technologies' in Berlin is gratefully acknowledged. We thank Rohit Saraf (Columbia University) for perceptive remarks.
} 
smiles and skews observed in the markets. As a remedy, various alternatives to the standard Libor market model have been proposed. They can be roughly categorized into three streams: local volatility models, stochastic volatility models, and jump-diffusion models. Especially jump-diffusion and stochastic volatility models are popular due to their economically meaningful behavior, and the greater flexibility they offer compared to local volatility models for instance. For local volatility Libor models we refer to Brigo and Mercurio (2006). Jump-diffusion models for assets go back to Merton (1976) and Eberlein (1998). Jamshidian (2001) developed a general semimartingale framework for the Libor process which covers the possibility of incorporating jumps as well as stochastic volatility. Specific jump-diffusion Libor models are proposed, among others, by Glasserman and Kou (2003) and Belomestny and Schoenmakers (2006). Levy Libor models are studied by Eberlein and Özkan (2005). Incorporation of stochastic volatility has been proposed by Andersen and Brotherton-Ratcliffe (2001), Piterbarg (2004), Wu and Zhang (2006), Zhu (2007). Recently, a Libor model with SABR stochastic volatility (Hagen et al. 2002) has attract some attention (Mercurio and Morini (2007)).

In the present article we focus on a flexible particularly structured Heston type stochastic volatility Libor model that, due to its very construction, can be calibrated to the cap/strike matrix in a robust way. In this model we incorporate a core idea from Belomestny and Schoenmakers (2006), who propose a jumpdiffusion Libor model as a perturbation of a given input Libor market model. As a main issue, Belomestny and Schoenmakers (2006) furnish the jump-diffusion extension in such a way that the (local) covariance structure of the extended model coincides with the (local) covariance structure of the market model. The approach of perturbing a given market model while preserving its covariance structure, has turned out to be fruitfull and is carried over into the design of the stochastic volatility Libor model presented in this paper. In fact, this idea is supported by the following arguments (see also Belomestny and Schoenmakers (2006)).

1. Cap(let) prices do not depend on the (local) correlation structure of forward Libors in a Libor market model but, typically, do depend (weakly) on it in a more general model. In contrast, swaption prices do depend significantly on this correlation structure. The Libor correlation structure may, for instance, be implied from a Libor market model calibration to prices of ATM swaptions (e.g. Brigo and Mercurio (2006), Schoenmakers (2005)). Therefore, we do not want to destroy this (input) correlation structure by setting it free while calibrating the extended model to the cap(let)-strike matrix.

2. The lack of smile behavior of a Libor market model is considered a consequence of Gaussianity of the driving random sources (Wiener processes). Therefore we want to perturb this Gaussian randomness to a non-Gaussian one by incorporating a CIR volatility process, while maintaining the (local) correlation structure of the Libor market model we started with. 
3. Preserving the correlation structure allows for robust calibration, since it significantly reduces the number of parameters to be calibrated while holding a realistic correlation structure.

Specifically, the perturbation part of the presented model will involve CIR (or square-root) volatility processes, and so the construction will finally resemble a Heston type Libor model (Heston (1993)). The CIR model originally derived in a framework based on equilibrium assumptions by Cox, Ingersoll, Ross (1985), is a special type affine process for which the characteristic function can be determined in closed form. For computing the characteristic function of fairly general affine processes with jump part we refer to recent work by Belomestny et al. (2008).

The idea of utilizing a Heston type process has already been formulated in Wu and Zhang (2006), and Zhu (2007). However, the present article differs from these works in the following respects.

1. As opposed to a one-dimensional stochastic volatility process as in $\mathrm{Wu} \&$ Zhang, or a (possibly) vector valued one which inhibits only one source of randomness as in Zhu (2007), we will study multi-dimensional CIR vector volatility processes with each component being driven by its own Brownian motion. This leads to a more realistic local correlation structure and renders the model more flexible for calibration.

2. We suggest a multi-dimensional partial-Gaussian and partial-Heston type model, where each forward Libor is driven by a linear combination of CIR processes.

3. While in both papers the issue of calibration has not been addressed, we give consideration to this problem using novel ideas mentioned above.

4. The new approach proposed in this paper may cure the limitations of known single volatility approaches, and, we show that a multiple stochastic volatility model must not be 'too complicated' as suggested in the literature (Piterbarg (2005)).

Furthermore, approximative analytic pricing formulas for caplets and swaptions are derived for this new Libor model which allow for fast calibration to these products. Ultimately, complex structured Over The Counter products may be priced by Monte Carlo using guidelines for simulating Heston type models as given in Kahl and Jäckel (2006).

The content of the paper is as follows. The multiple stochastic volatility extension of a (given) Libor model is introduced in Section 2. In Section 3 we outline a natural structuring of the model parameters, including the covariance constraint and some time homogeneity considerations. Section 4 deals with the Libor dynamics under different measures and prepares the tools for pricing and calibration to caps (Section 5) and pricing of swaptions (Section 6). A real life case study in Section 7 concludes. 


\section{Stochastic volatility extension of the Libor mar- ket model}

\subsection{The general Libor model}

Consider a fixed sequence of tenor dates $0=$ : $T_{0}<T_{1}<\ldots<T_{n}$, called a tenor structure, together with a sequence of so called day-count fractions $\delta_{i}:=T_{i+1}-T_{i}, i=1, \ldots, n-1$. With respect to this tenor structure we consider zero bond processes $B_{i}, i=1, \ldots, n$, where each $B_{i}$ lives on the interval $\left[0, T_{i}\right]$ and ends up with its face value $B_{i}\left(T_{i}\right)=1$. With respect to this bond system we deduce a system of forward rates, called Libor rates, which are defined by

$$
L_{i}(t):=\frac{1}{\delta_{i}}\left(\frac{B_{i}(t)}{B_{i+1}(t)}-1\right), \quad 0 \leq t \leq T_{i}, 1 \leq i<n .
$$

Note that $L_{i}$ is the annualized effective forward rate to be contracted at the date $t$, for a loan over a forward period $\left[T_{i}, T_{i+1}\right]$. Based on this rate one has to pay at $T_{i+1}$ an interest amount of $\$ \delta_{i} L_{i}\left(T_{i}\right)$ on a $\$ 1$ notional.

For a pre-specified volatility process $\gamma_{i} \in \mathbb{R}^{m}$, adapted to the filtration generated by some standard Brownian motion $W \in \mathbb{R}^{m}$, the dynamics of the corresponding Libor model have the form,

$$
\frac{d L_{i}}{L_{i}}=(\ldots) d t+\gamma_{i}^{\top} d W
$$

$i=1, \ldots, n-1$. The drift term, adumbrated by the dots, is known under different numeraire measures, such as the risk-neutral, spot, terminal and all measures induced by individual bonds taken as numeraire. If the processes $t \rightarrow \gamma_{i}(t)$ in (1) are deterministic, one speaks of a Libor market model.

\subsection{Extending the Libor market model}

In this work we study an extension of a generic Libor market model, which is given via a deterministic volatility structure $\gamma$. In particular, with respect to an extended Brownian filtration, we consider the following structure,

$$
\begin{aligned}
& \frac{d L_{i}}{L_{i}}=(\ldots) d t+\sqrt{1-r_{i}^{2}} \gamma_{i}^{\top} d W+r_{i} \beta_{i}^{\top} d U, \quad 1 \leq i<n, \\
& d U_{k}=\sqrt{v_{k}} d \widetilde{W}_{k} \quad 1 \leq k \leq d \\
& d v_{k}=\kappa_{k}\left(\theta_{k}-v_{k}\right) d t+\sigma_{k} \sqrt{v_{k}}\left(\rho_{k} d \widetilde{W}_{k}+\sqrt{1-\rho_{k}^{2}} d \bar{W}_{k}\right),
\end{aligned}
$$

where $\widetilde{W}$ and $\bar{W}$ are mutually independent $d$-dimensional standard Brownian motions, both independent of $W$. The coefficients $\beta_{i} \in \mathbb{R}^{d}$ in (2) are chosen to be deterministic vector functions. They will be specified later. The $r_{i}$ are constants 
that may be considered "allotment" or "proportion" factors, quantifying how much of the original input market model should be in play. For $r_{i}=0$ for all $i$, it is easily seen from (2) that the classical market model is retrieved. As such, at nonzero values of the $r_{i}$, the extended model may be regarded as a perturbation of the former. Finally, from a modeling point of view system (2) is obviously overparameterized in the following sense. By setting $\beta_{i k}=: \alpha_{k} \widetilde{\beta}_{i k}$ and $v_{k}=: \alpha_{k}^{-2} \widetilde{v}_{k}, \theta_{k}=: \alpha_{k}^{-2} \widetilde{\theta}_{k}, \sigma_{k}=: \alpha_{k}^{-1} \widetilde{\sigma}_{k}$, we retrieve exactly the same model. From now on we therefore normalize by setting $\theta_{k} \equiv 1$ without loss of generality.

It is helpful to think of the Libor model as a vector-valued stochastic process of dimension $n-1$ driven by $m+2 d$ standard Brownian motions with dynamics of the form

$$
\frac{d L_{i}}{L_{i}}=(\ldots) d t+\Gamma_{i}^{\top} d \mathcal{W}, \quad i=1, \ldots, n-1,
$$

where

$$
\Gamma_{i}=\left(\begin{array}{c}
\sqrt{1-r_{i}^{2}} \gamma_{i 1} \\
\cdot \\
\cdot \sqrt{1-r_{i}^{2}} \gamma_{i m} \\
r_{i} \beta_{i 1} \sqrt{v_{1}} \\
\cdot \\
\cdot \\
r_{i} \beta_{i d} \sqrt{v_{d}}
\end{array}\right) \quad d \mathcal{W}=\left(\begin{array}{c}
d W_{1} \\
\cdot \\
\cdot \\
d W_{m} \\
\widetilde{d W_{1}} \\
\cdot \\
\cdot \\
d \widetilde{W}_{d}
\end{array}\right)
$$

In (4) the square-root processes $v_{k}$ are given by (3) (with $\theta_{k} \equiv 1$ ).

In our approach we will work throughout under the terminal measure $P_{n}$. Following Jamshidian $(1997,2001)$, the Libor dynamics in this measure are given by

$$
\frac{d L_{i}}{L_{i}}=-\sum_{j=i+1}^{n-1} \frac{\delta_{j} L_{j}}{1+\delta_{j} L_{j}}\left(\sum_{k=1}^{m+d} \Gamma_{j k} \Gamma_{i k}\right) d t+\Gamma_{i}^{\top} d \mathcal{W}^{(n)}
$$

Often it turns out technically more convenient to work with the log-Libor dynamics. A straightforward application of Itô's lemma to (5) yields,

$$
d \ln L_{i}=-\frac{1}{2}\left|\Gamma_{i}\right|^{2} d t-\sum_{j=i+1}^{n-1} \frac{\delta_{j} L_{j}}{1+\delta_{j} L_{j}}\left(\sum_{k=1}^{m+d} \Gamma_{j k} \Gamma_{i k}\right) d t+\Gamma_{i}^{\top} d \mathcal{W}^{(n)}, \quad 1 \leq i<n .
$$




\section{Structuring the stochastic volatility extension}

\subsection{Covariance preservation of the market model}

Let us integrate the diffusion part of (6) and consider the resulting zero-mean random variable by

$$
\xi_{i}(t):=\int_{0}^{t} \Gamma_{i}^{\top} d \mathcal{W}^{(n)}
$$

For the covariance function of $\xi_{i}(t)$ in the terminal measure we have

$$
\begin{aligned}
E_{n}\left(\xi_{i}(t) \xi_{j}(t)\right) & =\sqrt{1-r_{i}^{2}} \sqrt{1-r_{j}^{2}} \int_{0}^{t} \gamma_{i}^{\top} \gamma_{j} d s+r_{i} r_{j} E_{n} \int_{0}^{t} \beta_{i}^{\top} d U \cdot \int_{0}^{t} \beta_{j}^{\top} d U \\
& =\sqrt{1-r_{i}^{2}} \sqrt{1-r_{j}^{2}} \int_{0}^{t} \gamma_{i}^{\top} \gamma_{j} d s+r_{i} r_{j} \sum_{k=1}^{d} E_{n} \int_{0}^{t} \beta_{i k} \beta_{j k} d\left\langle U_{k}\right\rangle \\
& =\sqrt{1-r_{i}^{2}} \sqrt{1-r_{j}^{2}} \int_{0}^{t} \gamma_{i}^{\top} \gamma_{j} d s+r_{i} r_{j} \sum_{k=1}^{d} \int_{0}^{t} \beta_{i k} \beta_{j k} E_{n} v_{k} d s \\
& =: \sqrt{1-r_{i}^{2}} \sqrt{1-r_{j}^{2}} \int_{0}^{t} \gamma_{i}^{\top} \gamma_{j} d s+r_{i} r_{j} \int_{0}^{t} \beta_{i}^{\top} \Lambda(t) \beta_{j} d s
\end{aligned}
$$

where $\Lambda(t)$ denotes a diagonal matrix in $\mathbb{R}^{d \times d}$ whose elements are the expected values $\lambda_{k}=E_{n} v_{k} \in \mathbb{R}$.

The square-root diffusions in (3) have a limiting stationary distribution. The transition law of the general CIR process,

$$
v(t)=v(u)+\int_{u}^{t}(\kappa(\theta-v(s)) d s+\sigma \sqrt{v(s)} d W(s)),
$$

is known. In particular, we have the representation

$$
v(t)=\frac{\sigma^{2}\left(1-e^{-\kappa(t-u)}\right)}{4 \kappa} \chi_{\alpha, c}^{2}, \quad t>u,
$$

where $\chi_{\alpha, c}^{2}$ is a noncentral chi-square random variable with $\alpha$ degrees of freedom and noncentrality $c$, where

$$
\alpha:=\frac{4 \theta \kappa}{\sigma^{2}}, \quad c:=\frac{4 \kappa e^{-\kappa(t-u)}}{\sigma^{2}\left(1-e^{-\kappa(t-u)}\right)} v(u) .
$$

For the expectation we have

$$
E\left[v(t) \mid \mathcal{F}_{u}\right]=(v(u)-\theta) e^{-\kappa(t-u)}+\theta, \quad t \geq u
$$

e.g. see Glasserman (2003) for details. So, it is natural to take the limit expectation as the starting value of the process. Thus, we set in (3)

$$
v_{k}(0)=\theta_{k}=1, \quad \text { for } \quad k=1, \ldots, d,
$$


to obtain $E v_{k}(t) \equiv 1$, hence $\Lambda=I$ is constant.

Recall that $\gamma_{i} \in \mathbb{R}^{m}$ is the (given) deterministic volatility structure of the input market model, for example obtained by some calibration procedure to ATM caps and ATM swaptions. We want to preserve the forward (log-)Libor covariance due to the structure $\gamma$ in some sense and now introduce the covariance constraint mentioned in the introduction. This restriction will be a modified version of the covariance restriction in Belomestny and Schoenmakers (2006) in fact. In the latter article one requires (in a jump-diffusion context)

$$
E_{n}\left(\xi_{i}(t) \xi_{j}(t)\right)=\int_{0}^{t} \gamma_{i}^{\top} \gamma_{j} d s \quad 1 \leq i, j<n .
$$

In view of (8) and (10), we set $r_{i} \equiv r$, to yield from (10),

$$
\int_{0}^{t} \beta_{i}^{\top} \beta_{j} d s=\int_{0}^{t} \gamma_{i}^{\top} \gamma_{j} d s, \quad 1 \leq i, j<n,
$$

which is obviously satisfied by taking $\beta \equiv \gamma$, and in particular $d=m$. In order to obtain closed-form expressions for characteristic functions of (log-)Libors later on, we need $\beta(t)$ to be piecewise constant in time, however. Therefore, as one possibility, we suggest to take

$$
\beta_{i}(t)=\gamma_{i}\left(T_{m(t)}\right), \quad \text { with } \quad m(t):=\inf \left\{j: T_{j} \geq t\right\}, \quad 0 \leq t \leq T_{i},
$$

such that (11) holds in a good approximation, as the integral is approximated by a Riemann sum in fact. If one strives for a more simple structure where $\beta$ is time-independent, we propose as a pragmatic choice, to take constant vectors $\beta_{i}$ according to

$$
\begin{aligned}
\beta_{i} & =\sigma_{i}^{\text {Black }} e_{i}, \quad \text { where } \\
\left(\sigma_{i}^{\text {Black }}\right)^{2} & :=\frac{1}{T_{i}} \int_{0}^{T_{i}}\left|\gamma_{i}(s)\right|^{2} d s, \\
e_{i}^{\top} e_{j} & :=\frac{\gamma_{i}^{\top} \gamma_{j}}{\left|\gamma_{i}\right|\left|\gamma_{j}\right|}(0)
\end{aligned}
$$

in order to match the covariance constraint (10) roughly. The requirement (13) may be considered as a relaxation of (11). Note that even when $m<n-1$, matching of (11) may require $d=n-1$. Depending on the readers preferences however, one may choose any $d, d<n-1$, and then fit (11) after dimension reduction via principal component analysis of the respective right-hand-sides.

\subsection{Time shift homogeneity}

From an economical point of view it is appealing to have a time shift homogeneous Libor dynamics. That is, the conditional distribution of $\left(L_{k+p}, L_{k+p+1}\right.$, ...) $\left(T_{k+p}\right)$ given the Libor state at $T_{k}(k, p>0)$ is the same as the conditional distribution of $\left(L_{p}, L_{p+1}, \ldots\right)\left(T_{p}\right)$ given the state at $T_{0}$. For a Libor market 
model this requirement is fulfilled when the deterministic volatility structure $\gamma$ satisfies $\gamma_{i}(t)=\gamma\left(T_{i}-t\right)=: g\left(T_{i}-t\right) e\left(T_{i}-t\right)$, where $g=|\gamma|$ and $e(s)$ is a (time dependent) unit vector. In practice it is not easy to identify such a unit vector function however. In an implementation it is much more convenient to work with piecewise constant (or even constant) unit vectors, for example of the form $e_{i-m(t)}$ with $m(t)$ as in (12) for a set of constant unit vectors $e_{i}$. On the other hand, it is well known that strict time shift homogeneity in the standard market model may lead to caplet fitting problems when market caplet volatilities decrease too fast in some sense (for details on market model calibration see for example Brigo and Mercurio (2006), and Schoenmakers (2005)). Altogether, it is reasonable to strive for time shift homogeneity as far as possible, both from a modeling and practical point of view. In this respect, it is recommendable to depart from an input Libor market model with a (nearly) time shift homogeneous volatility structure $\gamma$. Interestingly, if $\beta$ is then taken according to (12) in order to preserve covariance, $\beta$ will be nearly time shift homogeneous as well. For the more simple choice, constant $\beta$ according to (13), the extended Libor model (6) will still be close to time homogeneous. Therefore, and for simplicity, we deal in this paper only with the case of time-independent $\beta$, which satisfies (13).

\section{Dynamics under various measures}

\subsection{Dynamics under forward measures}

So far the Libor dynamics have been considered under the terminal measure. In order to price caplets later on, however, we will need to represent the above process under various forward measures. Let us denote the (time independent) solution of (13) by $\bar{\gamma} \in \mathbb{R}^{(n-1) \times d}$. Consequently spelling out (5) under the measure $P_{n}$ with $r_{i} \equiv r$ yields

$$
\begin{aligned}
\frac{d L_{i}}{L_{i}}= & -\sum_{j=i+1}^{n-1} \frac{\delta_{j} L_{j}}{1+\delta_{j} L_{j}}\left[\left(1-r^{2}\right) \gamma_{i}^{\top} \gamma_{j}+r^{2} \sum_{k=1}^{d} \bar{\gamma}_{i k} \bar{\gamma}_{j k} v_{k}\right] d t \\
& +\sqrt{1-r^{2}} \gamma_{i}^{\top} d W^{(n)}+r \sum_{k=1}^{d} \sqrt{v_{k}} \bar{\gamma}_{i k} d \widetilde{W}_{k}^{(n)}
\end{aligned}
$$

with corresponding volatility processes

$$
d v_{k}=\kappa_{k}\left(1-v_{k}\right) d t+\sigma_{k} \sqrt{v_{k}}\left(\rho_{k} d \widetilde{W}_{k}^{(n)}+\sqrt{1-\rho_{k}^{2}} d \bar{W}_{k}^{(n)}\right)
$$


By rearranging terms we may write,

$$
\begin{aligned}
\frac{d L_{i}}{L_{i}}= & \sqrt{1-r^{2}} \gamma_{i}^{\top}\left(d W^{(n)}-\sqrt{1-r^{2}} \sum_{j=i+1}^{n-1} \frac{\delta_{j} L_{j}}{1+\delta_{j} L_{j}} \gamma_{j} d t\right) \\
& +r \sum_{k=1}^{d} \bar{\gamma}_{i k} \sqrt{v_{k}}\left(d \widetilde{W}_{k}^{(n)}-r \sum_{j=i+1}^{n-1} \frac{\delta_{j} L_{j}}{1+\delta_{j} L_{j}} \bar{\gamma}_{j k} \sqrt{v_{k}} d t\right) \\
= & \sqrt{1-r^{2}} \gamma_{i}^{\top} d W^{(i+1)}+r \sum_{k=1}^{d} \bar{\gamma}_{i k} \sqrt{v_{k}} d \widetilde{W}_{k}^{(i+1)} .
\end{aligned}
$$

Since $L_{i}$ is a martingale under $P_{i+1}$, we have that both $W^{(i+1)}$ and $\widetilde{W}^{(i+1)}$ in (17) are standard Brownian motions under $P_{i+1}$. In terms of these new Brownian motions the volatility dynamics are

$$
\begin{aligned}
d v_{k}= & \kappa_{k}\left(1-v_{k}\right) d t+r \sigma_{k} \rho_{k} \sum_{j=i+1}^{n-1} \frac{\delta_{j} L_{j}}{1+\delta_{j} L_{j}} \bar{\gamma}_{j k} v_{k} d t \\
& +\rho_{k} \sigma_{k} \sqrt{v_{k}} d \widetilde{W}_{k}^{(i+1)}+\sqrt{1-\rho_{k}^{2}} \sigma_{k} \sqrt{v_{k}} d \bar{W}_{k}^{(n, i+1)} .
\end{aligned}
$$

As shown in the Appendix, the process $\bar{W}^{(n, i+1)}$ in (18) is a standard Brownian motion under both measures $P_{i+1}$ and $P_{n}$.

By freezing the Libors at their initial values in (18), we obtain approximative CIR dynamics

$$
d v_{k} \approx \kappa_{k}^{(i+1)}\left(\theta_{k}^{(i+1)}-v_{k}\right) d t+\sigma_{k} \sqrt{v_{k}}\left(\rho_{k} d \widetilde{W}_{k}^{(i+1)}+\sqrt{1-\rho_{k}^{2}} d \bar{W}_{k}^{(i+1)}\right)
$$

with reversion speed parameter

$$
\kappa_{k}^{(i+1)}:=\kappa_{k}-r \sigma_{k} \rho_{k} \sum_{j=i+1}^{n-1} \frac{\delta_{j} L_{j}(0)}{1+\delta_{j} L_{j}(0)} \bar{\gamma}_{j k}
$$

and mean reversion level

$$
\theta_{k}^{(i+1)}:=\frac{\kappa_{k}}{\kappa_{k}^{(i+1)}} .
$$

The approximative dynamics (19) for the volatility process will be used for calibration in Section 5 .

\subsection{Dynamics under swap measures}

An interest rate swap is a contract to exchange a series of floating interest payments in return for a series of fixed rate payments. Consider a series of 
payment dates between $T_{p+1}$ and $T_{q}, q>p$. The fixed leg of the swap pays $\delta_{j} K$ at each time $T_{j+1}, j=p, \ldots, q-1$, where $\delta_{j}=T_{j+1}-T_{j}$. In return, the floating leg pays $\delta_{j} L_{j}\left(T_{j}\right)$ at time $T_{j+1}$, where $L_{j}\left(T_{j}\right)$ is the rate fixed at time $T_{j}$ for payment at $T_{j+1}$. Consequently the time $t$ value of the interest rate swap is

$$
\sum_{j=p}^{q-1} \delta_{j} B_{j+1}(t)\left(L_{j}(t)-K\right) .
$$

The swap rate $S_{p, q}(t)$ is the value of the fixed rate $K$, such that the present value of the contract is zero, hence after some rearranging

$$
S_{p, q}(t)=\frac{\sum_{j=p}^{q-1} \delta_{j} B_{j+1}(t) L_{j}(t)}{\sum_{j=p}^{q-1} \delta_{j} B_{j+1}(t)}=\frac{B_{p}(t)-B_{q}(t)}{\sum_{j=p}^{q-1} \delta_{j} B_{j+1}(t)} .
$$

So $S_{p, q}$ is a martingale under the probability measure $P_{p, q}$, induced by the annuity numeraire $B_{p, q}=\sum_{j=p}^{q-1} \delta_{j} B_{j+1}(t)$. Therefore we may write

$$
d S_{p, q}(t)=\sigma_{p, q}(t) S_{p, q}(t) d \mathcal{W}^{(p, q)}(t),
$$

where $d \mathcal{W}^{(p, q)}(t)$ is standard Brownian motion under $P_{p, q}$. From (22) we see that the swap rate can be expressed as a weighted sum of the constituent forward rates,

$$
S_{p, q}(t)=\sum_{j=p}^{q-1} w_{j}(t) L_{j}(t)
$$

with

$$
w_{j}(t)=\frac{\delta_{j} B_{j+1}(t)}{B_{p, q}} .
$$

An application of Ito's Lemma yields

$$
\begin{aligned}
d S_{p, q}(t) & =\sum_{j=p}^{q-1} \frac{\partial S_{p, q}(t)}{\partial L_{j}(t)} d L_{j}(t)+\sum_{j=p}^{q-1} \sum_{i=p}^{q-1} \frac{\partial^{2} S_{p, q}}{\partial L_{j}(t) \partial L_{i}(t)} d L_{j}(t) d L_{i}(t) \\
& =\sum_{j=p}^{q-1} \frac{\partial S_{p, q}(t)}{\partial L_{j}(t)} L_{j}(t) \Gamma_{j}^{\top}\left[d \mathcal{W}^{(n)}+(\ldots) d t\right] .
\end{aligned}
$$

Equating (23) and (24), gives

$$
d S_{p, q}(t)=S_{p, q}(t)\left[\sum_{j=p}^{q-1} \nu_{j}(t) \Gamma_{j}^{\top}\right] d \mathcal{W}^{(p, q)}(t)
$$

with $\mathcal{W}^{(p, q)}=\left(W^{(p, q)}, \widetilde{W}^{(p, q)}\right)$ and

$$
\nu_{j}(t):=\frac{\partial S_{p, q}(t)}{\partial L_{j}(t)} \frac{L_{j}(t)}{S_{p, q}(t)} .
$$


The change of measure from $\mathcal{W}^{(n)}$ to $\mathcal{W}^{(p, q)}$ can be found in Schoenmakers (2005). In particular,

$$
d W^{(p, q)}=d W^{(n)}-\sqrt{1-r^{2}} \sum_{i=p}^{q-1} w_{i} \sum_{j=i+1}^{n-1} \frac{\delta_{j} L_{j}}{1+\delta_{j} L_{j}} \gamma_{j} d t
$$

and

$$
d \widetilde{W}_{k}^{(p, q)}=d \widetilde{W}_{k}^{(n)}-r \sum_{i=p}^{q-1} w_{i} \sum_{j=i+1}^{n-1} \frac{\delta_{j} L_{j}}{1+\delta_{j} L_{j}} \bar{\gamma}_{j k} \sqrt{v_{k}} d t .
$$

In terms of these new Brownian motions the volatility processes read

$$
\begin{aligned}
d v_{k}= & \kappa_{k}\left(1-v_{k}\right) d t+r \sigma_{k} \rho_{k} \sum_{i=p}^{q-1} w_{i}(t) \sum_{j=i+1}^{n-1} \frac{\delta_{j} L_{j}}{1+\delta_{j} L_{j}} \bar{\gamma}_{j k} v_{k} d t \\
& +\rho_{k} \sigma_{k} \sqrt{v_{k}} d \widetilde{W}_{k}^{(p, q)}+\sqrt{1-\rho_{k}^{2}} \sigma_{k} \sqrt{v_{k}} d \bar{W}_{k}^{(p, q, n)} .
\end{aligned}
$$

As shown in the Appendix, the process $\bar{W}^{(p, q, n)}$ in (25) is standard Brownian motion under both measures $P_{p, q}$ and $P_{n}$. Assuming now that $\frac{\partial S_{p, q}(t)}{\partial L_{j}(t)}$ and $\frac{L_{j}(t)}{S_{p, q}(t)}$ are approximately constant in time, we freeze the weights at their initial time $t=0$. Then the swap rate dynamic is approximately given by

$$
d S_{p, q}(t) \approx S_{p, q}(t)\left[\sum_{j=p}^{q-1} \nu_{j}(0) \Gamma_{j}^{\top}\right] d \mathcal{W}^{(p, q)}(t) .
$$

Similarly, freezing the Libors in the drift term of (25) leads to an approximated volatility process $v_{k}$ given by

$$
d v_{k} \approx \kappa_{k}^{(p, q)}\left(\theta_{k}^{(p, q)}-v_{k}\right) d t+\sigma_{k} \sqrt{v_{k}}\left(\rho_{k} d \widetilde{W}_{k}^{(p, q)}+\sqrt{1-\rho_{k}^{2}} d \bar{W}_{k}^{(p, q, n)}\right)
$$

with reversion speed parameter

$$
\kappa_{k}^{(p, q)}:=\kappa_{k}-r \sigma_{k} \rho_{k} \sum_{i=p}^{q-1} w_{i}(0) \sum_{j=i+1}^{n-1} \frac{\delta_{j} L_{j}(0)}{1+\delta_{j} L_{j}(0)} \bar{\gamma}_{j k},
$$

and mean reversion level

$$
\theta_{k}^{(p, q)}:=\frac{\kappa_{k}}{\kappa_{k}^{(p, q)}}
$$

\section{Pricing and calibration}

\subsection{Pricing caplets}

A caplet for the period $\left[T_{j}, T_{j+1}\right]$ with strike $K$ is an option that pays $\left(L_{j}\left(T_{j}\right)-\right.$ $K)^{+} \delta_{j}$ at time $T_{j+1}$, where $1 \leq j<n$. It is well-known that under the forward 
measure $P_{j+1}$ the $j$-th caplet price at time zero is given by

$$
C_{j}(K)=\delta_{j} B_{j+1}(0) E_{j+1}\left(L_{j}\left(T_{j}\right)-K\right)^{+} .
$$

Consequently under $P_{j+1}$ the $j$-th caplet price is determined by the dynamics of $L_{j}$ only. The FFT-method of Carr and Madan (1999) can be straightforwardly adapted to the caplet pricing problem as done in Belomestny and Schoenmakers (2006). We here recap the main results.

In terms of the log-moneyness variable

$$
v:=\ln \frac{K}{L_{j}(0)}
$$

the $j$-th caplet price can be expressed as

$$
\mathcal{C}_{j}(v):=C_{j}\left(e^{v} L_{j}(0)\right)=\delta_{j} B_{j+1}(0) L_{j}(0) E_{j+1}\left(e^{X_{j}\left(T_{j}\right)}-e^{v}\right)^{+},
$$

where $X_{j}(t)=\ln L_{j}(t)-\ln L_{j}(0)$. One then defines the auxiliary function

$$
\mathcal{O}_{j}(v):=\delta_{j}^{-1} B_{j+1}^{-1}(0) L_{j}^{-1}(0) \mathcal{C}_{j}(v)-\left(1-e^{v}\right)^{+}
$$

and can show the following proposition.

Proposition 1 For the Fourier transform of the function $\mathcal{O}_{j}$ defined above and $\varphi_{j+1}(\cdot ; t)$ denoting the characteristic function of the process $X_{j}(t)$ under $P_{j+1}$ we have

$$
\mathcal{F}\left\{\mathcal{O}_{j}\right\}(z)=\int_{-\infty}^{\infty} \mathcal{O}_{j}(v) e^{\mathfrak{i} v z} d v=\frac{1-\varphi_{j+1}\left(z-\mathfrak{i} ; T_{j}\right)}{z(z-\mathfrak{i})} .
$$

The proof can be found in Belomestny and Reiß (2006). Next, combining (30), (31), and (32) yields

$$
\begin{aligned}
C_{j}(K)= & \delta_{j} B_{j+1}(0)\left(L_{j}(0)-K\right)^{+} \\
& +\frac{\delta_{j} B_{j+1}(0) L_{j}(0)}{2 \pi} \int_{-\infty}^{\infty} \frac{1-\varphi_{j+1}\left(z-\mathfrak{i} ; T_{j}\right)}{z(z-\mathfrak{i})} e^{-\mathfrak{i} z \ln \frac{K}{L_{j}(0)}} d z .
\end{aligned}
$$

\subsection{Calibration road map}

We now outline a calibration procedure for the Libor structure (2), under the following additional assumptions.

(i) The input market Libor volatility structure $\gamma \in \mathbb{R}^{(n-1) \times m}$ is assumed to be of full rank, that is $m=n-1$. (Strictly speaking it would be enough to require the right-hand-side of (11) to be of full rank.)

(ii) The terminal log-Libor increment $d \ln L_{n-1}$ is influenced by a single stochastic volatility shock $d U_{n-1}$, the one but last, hence $d \ln L_{n-2}$, by only $d U_{n-1}$ and $d U_{n-2}$, and so forth. Put differently, we assume $\beta \in \mathbb{R}^{(n-1) \times d}$ to be a squared upper triangular matrix of rank $n-1$, hence $d=n-1$. 
(iii) The $r_{i}$ are taken to be constant, that is $r_{i} \equiv r$, and the matrix $\beta$ is determined as the time independent upper triangular solution $\bar{\gamma}$ of the covariance condition (13).

(iv) Recall that $v_{k}(0) \equiv \theta_{k} \equiv 1,1 \leq k<n$.

For the Libor dynamics structured in the above way we thus have

$$
\begin{aligned}
d \ln L_{i}(t)= & -\frac{1}{2}\left[\left(1-r^{2}\right)\left|\gamma_{i}\right|^{2}+r^{2} \sum_{k=i}^{n-1} \bar{\gamma}_{i k}^{2} v_{k}\right] d t \\
& +\sqrt{1-r^{2}} \gamma_{i}^{\top} d W^{(i+1)} \\
& +r \sum_{k=i}^{n-1} \bar{\gamma}_{i k} \sqrt{v_{k}} d \widetilde{W}_{k}^{(i+1)}, \quad 1 \leq i<n,
\end{aligned}
$$

where for $i=n-1$ the dynamics of $v_{n-1}$ is given by (16), and for $i<n-1$ the dynamics of $v_{k}, i \leq k<n$, is approximately given by (19).

We will calibrate the structure to prices of caplets according to the following roadmap.

1. First step $i=n-1$. Calibrate $r$ and the parameter set $\left(\kappa_{n-1}, \theta_{n-1}=\right.$ $\left.1, \sigma_{n-1}, \rho_{n-1}\right)$ to the $T_{n-1}$ column of the cap-strike matrix via (33) using the explicitly known characteristic function $\varphi_{n}$ of $\ln \left[L_{n-1}\left(T_{n-1}\right) / L_{n-1}(0)\right]$ (see Appendix (8.0.1)).

2. For $i=n-2$ down to 1 carry out the next iteration step:

3. The k-th step $i=n-k$. Transform the yet known parameter set $\left(\kappa_{j}, \sigma_{j}, \rho_{j}\right)$ $i<j<n$, via (20) and (21) into the corresponding set $\left(\kappa_{j}^{(i+1)}, \sigma_{j}^{(i+1)}, \rho_{j}^{(i+1)}, \theta_{j}^{(i+1)}\right), i<j<n$. By the upper triangular structure of the square matrix $\bar{\gamma}$ we obviously have $\kappa_{i}^{(i+1)}=\kappa_{i}$, hence by (21) $\theta_{i}^{(i+1)}=1$. Then calibrate the at this stage unknown parameter set $\left(\kappa_{i}, \sigma_{i}, \rho_{i}\right)$ to the $T_{i}$ column of the cap-strike matrix via (33) using the explicitly known characteristic function $\varphi_{i+1}$ of $\ln \left[L_{i}\left(T_{i}\right) / L_{i}(0)\right]$ under the approximation (17)-(19) (see Appendix (8.0.1)).

The above calibration algorithm includes at each step, as usual, the minimization of some objective function. As such function we take the weighted sum of squares of the corresponding differences between observed market prices and prices induced by the model. The weights are taken to be proportional to BlackScholes vegas. As initial values for the local optimization routine at time step $i+1$ the values of estimated parameters at time step $i$ are used. 


\section{Pricing swaptions}

A European swaption over a period $\left[T_{p}, T_{q}\right]$ gives the right to enter at $T_{p}$ into an interest rate swap with strike rate $K$. The swaption value at time $t \leq T_{p}$ is given by

$$
\operatorname{Swpn}_{p, q}(t)=B_{p, q}(t) E_{p, q}^{\mathcal{F}_{t}}\left(S_{p, q}\left(T_{p}\right)-K\right)^{+} .
$$

Since the approximative model (26)-(27) for $S_{p, q}$ has an affine structure with constant coefficients one can write down the characteristic function of $S_{p, q}$ analytically under $P_{p, q}$ and follow the lines of the previous section to calibrate the model.

Remark 2 Due to the covariance restrictions (11)-(13), one can expect that the model prices of ATM swaptions are not far from market prices because our model employs a covariance structure of LMM calibrated to the market prices of ATM swaptions.

\section{Calibration to real data: a first case study}

In this section we calibrate the model (17)-(19) to two caplet-strike volatility matrices available at the market on 19.06.2008 and 26.06.08 respectively, which are partially shown in Tables 1,2. A corresponding implied volatility surface is shown in Figure 2, where smiles are clearly observable. Due to the structure of the given data sets we consider a Libor model based on semi-annual tenors, i.e. $\delta_{j} \equiv 0.5$, with $n=41$ (20 years).

In a pre-calibration a standard market model is calibrated to ATM caps and ATM swaptions using Schoenmakers (2005). However, we emphasize that the method by which this input market model is obtained is not essential nor considered a discussion point for this paper. For the pre-calibration we have used a volatility structure of the form

$$
\gamma_{i}(t)=c_{i} g\left(T_{i}-t\right) e_{i+1-m(t)}, \quad 0<t \leq T_{i}, \quad 1 \leq i<n
$$

where $g$ is a simple parametric function, $e_{i}$ are unit vectors, and $m(t)$ is defined in (12). The pre-calibration routine returns $e_{i} \in \mathbb{R}^{n-1}$ such that $\left(e_{i, k}\right)$ is upper triangular and

$$
\begin{aligned}
e_{i}^{\top} e_{j}=\rho_{i j}= & \exp \left[-\frac{|j-i|}{m-1}\left(-\ln \rho_{\infty}\right.\right. \\
& \left.\left.-\eta \frac{i^{2}+j^{2}+i j-m i-m j-3 i-3 j+3 m+2}{(m-2)(m-3)}\right)\right], \\
& i, j=1, \ldots, m:=n-1,0 \leq \eta \leq-\ln \rho_{\infty} .
\end{aligned}
$$

The function $g$ is parameterized as

$$
g(s)=g_{\infty}+\left(1-g_{\infty}+a s\right) e^{-b s} .
$$


For the Libor market model the loading factors $c_{i}$ are readily computed from

$$
\left(\sigma_{T_{i}}^{A T M}\right)^{2} T_{i}=c_{i}^{2} \int_{0}^{T_{i}} g^{2}(s) d s, \quad i=1, \ldots, n-1 .
$$

The initial Libor curve, is directly obtained from present values given at the respective calibration dates and (partially) given in Table 3.

\section{Calibrating the market model}

The market model calibration is based on an objective function which involves the squared distance between a set of market and model swaption volatilities, and a term which penalizes the deviation $\sum_{i}\left(c_{i}-c_{i+1}\right)^{2}$ from being constant, where the $c_{i}$ s are computed from (37) (see also Section 3.2 for a motivation). For the respective dates Table 4 shows the parameters for the scalar volatility function (36) and correlation matrix (35) based on a calibration of the market model to 93 swaption quotes. These scalar volatility functions and correlation structures are taken as inputs for the stochastic volatility model while the constants $c_{i}$ will be calibrated newly for flexibility. The results of the calibration of the multiple stochastic volatility model to the cap-strike matrix at the respective calibration dates are given in Tables 5,6 . We note that the stochastic volatility calibration is done with respected option prices (rather than volatilities as usual when calibrating a market model).

\section{Comments on the calibration}

It turned that for these data sets the stochastic volatility parameter $r$ needed to be taken rather close to one, $r \equiv 0.9$. A qualitative impression of the calibration can be obtained from Figure 1. From the last down to the sixed tenor the relative average price calibration fit is about $5 \%$ for both data sets. For the short term tenors (up to the fifth) the calibration errors growth up to about 13-25\% unfortunately, and are therefore not reported. We found out however that the main reason for this bad fit for small maturities is the erratic behavior of the yield curve over this period at the calibration dates (see Table 3). For instance after replacing the actual yield curve with a smoothed one we also got a good fit for small maturities.

The overall relative root-mean-square fit we have reached shows to be $0.5 \%$ $5 \%$, when the caplet maturity ranges from 0.5 to 20 .

\section{Concluding remark}

We have proposed an economically motivated multiple stochastic volatility extension of a given (pre-calibrated) Libor market model which is suited for Monte Carlo simulation of exotic interest rate products. Also it is shown that this extension allows for fast (approximative) cap and swaption pricing with smiles which enables efficient calibration to these products. A road map for calibration to the cap-strike matrix is given and illustrated by a case study. The considered 
Figure 1: Caplet volas from the calibrated model (solid lines) and market caplets volas $\sigma_{T}^{K}$ (dashed lines) for different caplet periods.

data sets in this study were taken at rather turbulent times, to reveal some stress issues of the model calibration. We just note that by considering more smooth data sets (smooth yield curves in particular), it is observed that the calibration performs overall satisfactory. Finally, we underline that in this paper the main focus is on the structure of the presented stochastic volatility model and its implementation. An in-depth analysis of the model calibration and its performance, for instance analysis of more case studies, and calibration to other products such as CMS-spreads, is the subject of subsequent work (Belomestny, Kolodko, Schoenmakers (2008)).

\section{Appendix}

\subsubsection{The Conditional Characteristic Function}

For $j=1, \ldots, n-1$, we need to determine the characteristic function of $\ln L_{j}(T)-$ $\ln L_{j}(0)$ under the relevant measure $P_{j+1}$. For each component $k=1, \ldots, n-1$ the Heston CIR-process has the general form 


$$
d v_{k}=\kappa_{k}^{(j+1)}\left(\theta_{k}^{(j+1)}-v_{k}\right) d t+\sigma_{k} \rho_{k} \sqrt{v_{k}} d \widetilde{W}_{k}^{(j+1)}+\sigma_{k} \sqrt{\left(1-\rho_{k}^{2}\right)} \sqrt{v_{k}} d \bar{W}_{k}^{(j+1)}
$$

In this case and a forward Libor dynamic given by (34), with general $v \in \mathbb{R}^{n-1}$, the solution is of the form

$$
\begin{aligned}
\varphi_{j+1}(z ; T, l, v) & =E_{j+1}\left[e^{i z \ln \frac{L_{j}(T)}{L_{j}(0)}} \mid v_{k}(0)=v_{k}, k=1, \ldots, n-1\right] \\
& =\varphi_{j+1,0}(z ; T) \prod_{k=j}^{n-1} \varphi_{j+1, k}\left(z ; T, v_{k}\right)
\end{aligned}
$$

where

$$
\varphi_{j+1,0}(z ; T)=\exp \left(-\frac{1}{2}\left(1-r^{2}\right) \eta_{j}^{2}(T)\left(z^{2}+\mathfrak{i} z\right)\right), \quad \eta_{j}^{2}(T)=\int_{0}^{T}\left|\gamma_{j}\right|^{2} d t
$$

and for each fixed $k, \varphi_{j+1, k}\left(z ; T, v_{k}\right):=\widehat{p}_{j+1, k}\left(z ; T, y_{k}, v_{k}\right)_{y_{k}=0}$, where $\widehat{p}_{j+1, k}$ satisfies the parabolic equation

$$
\begin{aligned}
\frac{\partial \widehat{p}_{j+1, k}}{\partial T} & =\kappa_{k}^{(j+1)}\left(\theta_{k}^{(j+1)}-v_{k}\right) \frac{\partial \widehat{p}_{j+1, k}}{\partial v_{k}}-\frac{1}{2} r^{2} \bar{\gamma}_{j k}^{2} v_{k} \frac{\partial \widehat{p}_{j+1, k}}{\partial y_{k}}+\frac{1}{2} \sigma_{k}^{2} v_{k} \frac{\partial^{2} \widehat{p}_{j+1, k}}{\partial v_{k}^{2}} \\
& +\frac{1}{2} r^{2} \bar{\gamma}_{j k}^{2} v_{k} \frac{\partial^{2} \widehat{p}_{j+1, k}}{\partial y_{k}{ }^{2}}+\sigma_{k} \rho_{k} r \bar{\gamma}_{j k} v_{k} \frac{\partial^{2} \widehat{p}_{j+1, k}}{\partial y_{k} \partial v_{k}}
\end{aligned}
$$

with the boundary condition

$$
\widehat{p}_{j+1, k}\left(z ; 0, y_{k}, v_{k}\right)=e^{\mathfrak{i} z y_{k}},
$$

as can be easily verified by the Feynman-Kac formula. It is well known that the above equation can be solved explicitly by the ansatz

$$
\widehat{p}_{j+1, k}\left(z ; T, y_{k}, v_{k}\right)=\exp \left(A_{j, k}(z ; T)+v_{k} B_{j, k}(z ; T)+\mathfrak{i} z y_{k}\right),
$$

which yields a Riccati equation in $A_{j, k}$ and $B_{j, k}$ with solution

$$
\begin{aligned}
& A_{j, k}(z ; T)=\frac{\kappa_{k}^{(j+1)} \theta_{k}^{(j+1)}}{\sigma_{k}^{2}}\left\{\left(a_{j, k}-d_{j, k}\right) T-2 \ln \left[\frac{e^{-d_{j, k} T}-g_{j, k}}{1-g_{j, k}}\right]\right\} \\
& B_{j, k}(z ; T)=\frac{\left(a_{j, k}+d_{j, k}\right)\left(1-e^{d_{j, k} T}\right)}{\sigma_{k}^{2}\left(1-g_{j, k} e^{d_{j, k} T}\right)},
\end{aligned}
$$

where

$$
\begin{aligned}
a_{j, k} & =\kappa_{k}^{(j+1)}-\mathfrak{i} r \rho_{k} \sigma_{k} \bar{\gamma}_{j k} z \\
d_{j, k} & =\sqrt{a_{j, k}^{2}+r^{2} \bar{\gamma}_{j k}^{2} \sigma_{k}^{2}\left(z^{2}+\mathfrak{i} z\right)} \\
g_{j, k} & =\frac{a_{j, k}+d_{j, k}}{a_{j, k}-d_{j, k}} .
\end{aligned}
$$


We thus obtain

$$
\varphi_{j+1, k}\left(z ; T, v_{k}\right)=\exp \left(A_{j, k}(z ; T)+v_{k} B_{j, k}(z ; T)\right) .
$$

In (39) we have chosen the formulation of Lord and Kahl (2005) which has the convenient property that we can take in (39) for the complex logarithm always the principle branch. Note that the first lower index $j+1$ in the characteristic function refers to the measure, whereas the first index $j$ at the introduced coefficients refers to relevant forward Libor. The second index refers to the component.

It is again the choice of $\bar{\gamma}$ that enables the product in (38) to be startet at $j$. This crucial feature will show to be beneficial in the calibration part. When $j=n-1$, for example, only the last log-Libor will contribute a non-trivial factor to the characteristic function. For all others we have

$$
\varphi_{n, k} \equiv 1, \quad k=1, \ldots, n-2 .
$$

\subsubsection{CIR}

Consider a CIR model of the form,

$$
d v(t)=\kappa(\theta-v(t)) d t+\sigma \sqrt{v(t)} d W(t), \quad \kappa, \theta, \sigma>0 .
$$

Given $v(u), v(t)$ with $t>u$ is distributed with density

$$
\nu \chi_{d}^{2}(\nu x, \xi)
$$

where $\chi_{d}^{2}(x, \xi)$ is the density of a noncentral chi-square random variable with $d$ degrees of freedom and noncentrality parameter $\xi$ and

$$
\begin{aligned}
& \nu=\frac{4 \kappa}{\sigma^{2}\left(1-e^{-\kappa(t-u)}\right)} \\
& \xi=\frac{4 \kappa e^{-\kappa(t-u)}}{\sigma^{2}\left(1-e^{-\kappa(t-u)}\right)} v(u) \\
& d=\frac{4 \theta \kappa}{\sigma^{2}} .
\end{aligned}
$$

The conditional mean of $v(t)$ is given by

$$
E(v(t) \mid v(u))=\nu^{-1}(\xi+d)=(v(u)-\theta) e^{-\kappa(t-u)}+\theta
$$

and the conditional second moment is

$$
\begin{aligned}
E\left(v^{2}(t) \mid v(u)\right) & =\frac{\left(2(d+2 \xi)+(\xi+d)^{2}\right)}{\nu^{2}} \\
& =\left(1+\frac{2}{d}\right)[E(v(t) \mid v(u))]^{2}-\frac{2}{d} e^{-2 \kappa(t-u)} v^{2}(u) .
\end{aligned}
$$




\subsubsection{Measure Invariance}

Why is $d \bar{W}_{k}^{(n, i+1)}$ invariant under the various measures?

See Jamshidian for the compensator, which is given by

$$
\mu_{\bar{W}_{k}^{(n)}}^{i+1}=\left\langle\bar{W}_{k}^{(n)}, \ln M\right\rangle .
$$

with

$$
M=\Pi_{j=i+1}^{n-1}\left(1+\delta_{j} L_{j}\right) .
$$

That is, we have

$$
\begin{aligned}
\left\langle\bar{W}_{k}^{(n)}, \ln M\right\rangle & =d \bar{W}_{k}^{(n)} d \ln M=d \bar{W}_{k}^{(n)} d\left(\sum_{j=i+1}^{n-1} \ln \left(1+\delta_{j} L_{j}\right)\right) \\
& =\sum_{j=i+1}^{n-1} d \bar{W}_{k}^{(n)} d \ln \left(1+\delta_{j} L_{j}\right) \\
& =\sum_{j=i+1}^{n-1} \frac{\delta_{j} L_{j}}{1+\delta_{j} L_{j}} d \bar{W}_{k}^{(n)} d \ln L_{j}
\end{aligned}
$$

A closer look at (15) reveils that all terms are negligible, since of higher order than $d t$, or zero due to independence of $\bar{W}$ and $W$ or $\widetilde{W}$, respectively. We thus have

$$
\left\langle\bar{W}_{k}^{(n)}, \ln M\right\rangle=0
$$

or in other words, as indicated by $d \bar{W}_{k}^{(n, i+1)}$ :

$$
d \bar{W}_{k}^{(n)}=d \bar{W}_{k}^{(i+1)} .
$$

Analogously we obtain by exchanging $\bar{W}_{k}$ with $\widetilde{W}_{k}$ that

$$
\begin{aligned}
\left\langle\widetilde{W}_{k}^{(n)}, \ln M\right\rangle & =d \widetilde{W}_{k}^{(n)} d \ln M \\
& =\sum_{j=i+1}^{n-1} \frac{\delta_{j} L_{j}}{1+\delta_{j} L_{j}} d \widetilde{W}_{k}^{(n)} d \ln L_{j} \\
& =\sum_{j=i+1}^{n-1} \frac{r \delta_{j} L_{j}}{1+\delta_{j} L_{j}} \beta_{j k} \sqrt{v_{t}^{k}} d t .
\end{aligned}
$$




\section{References}

[1] Andersen, L. and R. Brotherton-Ratcliffe (2001). Extended Libor Market Models with Stochastic Volatility. Working paper, Gen Re Securities.

[2] Andersen, L. and Piterbarg, V. (2007). Moment Explosions in Stochastic Volatility Models. Finance Stoch. 11, no. 1, 29-50.

[3] Belomestny, D., Kampen, J. and J. Schoenmakers (2008). Holomorphic transforms with application to affine processes. WIAS preprint 1297.

[4] Belomestny, D., Kolodko, A. and J. Schoenmakers (2008). Pricing of CMS spread options in a multiple stochastic volatility Libor model. Working paper.

[5] Belomestny, D. and M. Reiß (2006). Optimal calibration of exponential Lévy models. Finance Stoch. 10, no. 449-474, 29-50.

[6] Belomestny, D. and J.G.M. Schoenmakers (2006). A Jump-Diffusion Libor Model and its Robust Calibration, Preprint No. 1113, WIAS Berlin.

[7] Brigo, D. and F. Mercurio (2001) Interest rate models - theory and practice. Springer Finance. Springer-Verlag, Berlin.

[8] Brace, A., Gatarek, D. and M. Musiela (1997). The Market Model of Interest Rate Dynamics. Mathematical Finance, 7 (2), 127-155.

[9] Carr, P. and D. Madan (1999). Option Valuation Using the Fast Fourier Transform, Journal of Computational Finance, 2, 61Ū74.

[10] Cox, J.C., Ingersoll, J.E. and S.A. Ross (1985). A Theory of the Term Structure of Interest Rates, Econometrica 53, 385-407.

[11] Eberlein, E., Keller U. and K. Prause (1998). New insights into smile, mispricing, and value at risk: the hyperbolic model. Journal of Business, 71(3), 371Ü405.

[12] Eberlein, E. and F. Özkan (2005). The Lévy Libor model, Finance Stoch. 7, no. 1, 1-27.

[13] Glasserman, P. Monte Carlo methods in financial engineering. Applications of Mathematics (New York), 53. Stochastic Modelling and Applied Probability. Springer-Verlag, New York, 2004.

[14] Glasserman, P. and S.G. Kou (2003). The term structure of simple forward rates with jump risk. Mathematical Finance 13, no. 3, 383-410.

[15] Hagan, P. S., Kumar, D., Lesniewski, A. S. and D. E. Woodward (2002). 'Managing smile risk', WILMOTT Magazine September, 84-108. 
[16] Heston, S. (1993). A closed-form solution for options with stochastic volatility with applications to bond and currency options. The Review of Financial Studies, Vol. 6, No. 2, 327-343.

[17] Jamshidan, F.(1997). LIBOR and swap market models and measures. Finance and Stochastics, 1, 293-330.

[18] Jamshidian, F.(2001). LIBOR Market Model with Semimartingales, in "Option Pricing, Interest Rates and Risk Management", Cambridge Univ.

[19] Kahl Ch. and P. Jäckel (2006). Fast strong approximation Monte Carlo schemes for stochastic volatility models. Quantitative Finance, 6(6), 513536.

[20] Lord R. and C. Kahl (2005). Complex logarithms in Heston-like models. working paper to appear in Math. Fin.

[21] Mercurio M. and M. Morini (2007). No-arbitrage dynamics for a tractable SABR term structure Libor Model. SSRN Working Paper.

[22] Merton, R.C. (1976). Option pricing when underlying stock returns are discontinuous. J. Financial Economics, 3(1), 125-144.

[23] Miltersen, K., K. Sandmann, and D. Sondermann (1997). Closed-form solutions for term structure derivatives with lognormal interest rates. Journal of Finance, 409-430.

[24] Piterbarg, V. (2004). A stochastic volatility forward Libor model with a term structure of volatility smiles. SSRN Working Paper.

[25] Piterbarg, V. (2005). Is CMS spread volatility sold too cheap? Presented at II Fixed Income Conference, Prague.

[26] Schoenmakers, J.: Robust Libor Modelling and Pricing of Derivative Products. Boca Raton London New York Singapore: Chapman \& Hall - CRC Press 2005

[27] Wu, L. and F. Zhang (2006). Libor Market Model with Stochastic Volatility. Journal of Industrial and Management Optimization, 2, 199-207.

[28] Zhu, J. (2007). An extended Libor Market Model with nested stochastic volatility dynamics. SSRN Working Paper. 


\begin{tabular}{|c|cccccc|}
\hline $\mathrm{T} / \mathrm{K}$ & 2.00 & 3.00 & 4.00 & 5.00 & 6.00 & 8.00 \\
\hline \hline 1 & 0.325 & 0.244 & 0.19 & 0.165 & 0.174 & 0.22 \\
\hline 1.5 & 0.372 & 0.295 & 0.237 & 0.196 & 0.198 & 0.223 \\
\hline 2 & 0.374 & 0.299 & 0.246 & 0.208 & 0.205 & 0.224 \\
\hline 3 & 0.347 & 0.283 & 0.241 & 0.213 & 0.205 & 0.212 \\
\hline 4 & 0.325 & 0.266 & 0.228 & 0.204 & 0.196 & 0.201 \\
\hline 5 & 0.307 & 0.252 & 0.217 & 0.196 & 0.189 & 0.192 \\
\hline 6 & 0.294 & 0.241 & 0.208 & 0.189 & 0.182 & 0.184 \\
\hline 7 & 0.283 & 0.232 & 0.201 & 0.183 & 0.176 & 0.176 \\
\hline 8 & 0.274 & 0.225 & 0.194 & 0.177 & 0.17 & 0.169 \\
\hline 9 & 0.267 & 0.219 & 0.189 & 0.172 & 0.164 & 0.162 \\
\hline 10 & 0.262 & 0.215 & 0.184 & 0.167 & 0.159 & 0.156 \\
\hline 12 & 0.251 & 0.206 & 0.177 & 0.16 & 0.151 & 0.147 \\
\hline 15 & 0.238 & 0.195 & 0.167 & 0.151 & 0.142 & 0.137 \\
\hline 20 & 0.226 & 0.184 & 0.157 & 0.141 & 0.133 & 0.13 \\
\hline
\end{tabular}

Table 1: Subset out of 195 caplet volatilities $\sigma_{T}^{K}$ (in \%) for different strikes and different tenor dates (in years), 19.06.2008. 


\begin{tabular}{|c|cccccc|}
\hline $\mathrm{T} / \mathrm{K}$ & 2.00 & 3.00 & 4.00 & 5.00 & 6.00 & 8.00 \\
\hline \hline 1 & 0.305 & 0.23 & 0.18 & 0.158 & 0.167 & 0.209 \\
\hline 1.5 & 0.353 & 0.281 & 0.228 & 0.19 & 0.195 & 0.223 \\
\hline 2 & 0.355 & 0.285 & 0.236 & 0.201 & 0.202 & 0.225 \\
\hline 3 & 0.34 & 0.274 & 0.231 & 0.206 & 0.203 & 0.22 \\
\hline 4 & 0.327 & 0.261 & 0.221 & 0.199 & 0.197 & 0.213 \\
\hline 5 & 0.313 & 0.25 & 0.212 & 0.192 & 0.19 & 0.204 \\
\hline 6 & 0.297 & 0.239 & 0.204 & 0.186 & 0.182 & 0.191 \\
\hline 7 & 0.283 & 0.23 & 0.198 & 0.18 & 0.175 & 0.18 \\
\hline 8 & 0.273 & 0.223 & 0.192 & 0.174 & 0.168 & 0.17 \\
\hline 9 & 0.265 & 0.217 & 0.187 & 0.169 & 0.162 & 0.162 \\
\hline 10 & 0.259 & 0.212 & 0.182 & 0.165 & 0.157 & 0.156 \\
\hline 12 & 0.248 & 0.203 & 0.175 & 0.158 & 0.149 & 0.145 \\
\hline 15 & 0.235 & 0.193 & 0.166 & 0.149 & 0.139 & 0.134 \\
\hline 20 & 0.223 & 0.183 & 0.156 & 0.14 & 0.13 & 0.126 \\
\hline
\end{tabular}

Table 2: Subset out of 195 caplet volatilities $\sigma_{T}^{K}$ (in \%) for different strikes and different tenor dates (in years), 26.06.2008. 
Figure 2: Caplet implied volatility surface $\sigma_{T}^{K}$. 


\begin{tabular}{|r|c|c||r|c|c|}
\hline$T_{i}$ & $L_{i}(0) 19.06 .08$ & $L_{i}(0) 26.06 .08$ & $T_{i}$ & $L_{i}(0) 19.06 .08$ & $L_{i}(0) 26.06 .08$ \\
\hline \hline 0.5 & 0.0582 & 0.0587 & 10.5 & 0.0500 & 0.0516 \\
\hline 1 & 0.0665 & 0.0669 & 11 & 0.0500 & 0.0520 \\
\hline 1.5 & 0.0514 & 0.0500 & 11.5 & 0.0502 & 0.0522 \\
\hline 2 & 0.0390 & 0.0368 & 12 & 0.0504 & 0.0523 \\
\hline 2.5 & 0.0476 & 0.0461 & 12.5 & 0.0503 & 0.0520 \\
\hline 3 & 0.0557 & 0.0561 & 13 & 0.0502 & 0.0520 \\
\hline 3.5 & 0.0517 & 0.0520 & 13.5 & 0.0502 & 0.0521 \\
\hline 4 & 0.0472 & 0.0471 & 14 & 0.0501 & 0.0521 \\
\hline 4.5 & 0.0475 & 0.0477 & 14.5 & 0.0500 & 0.0518 \\
\hline 5 & 0.0481 & 0.0488 & 15 & 0.0498 & 0.0517 \\
\hline 5.5 & 0.0474 & 0.0485 & 15.5 & 0.0496 & 0.0517 \\
\hline 6 & 0.0466 & 0.0484 & 16 & 0.0494 & 0.0515 \\
\hline 6.5 & 0.0473 & 0.0488 & 16.5 & 0.0491 & 0.0510 \\
\hline 7 & 0.0477 & 0.0493 & 17 & 0.0489 & 0.0508 \\
\hline 7.5 & 0.0480 & 0.0497 & 17.5 & 0.0488 & 0.0509 \\
\hline 8 & 0.0484 & 0.0500 & 18 & 0.0487 & 0.0508 \\
\hline 8.5 & 0.0489 & 0.0504 & 18.5 & 0.0485 & 0.0503 \\
\hline 9 & 0.0493 & 0.0508 & 19 & 0.0482 & 0.0498 \\
\hline 9.5 & 0.0497 & 0.0511 & 19.5 & 0.0479 & 0.0495 \\
\hline 10 & 0.0499 & 0.0514 & 20 & 0.0476 & 0.0493 \\
\hline
\end{tabular}

Table 3: Initial Libor curves

\begin{tabular}{|c||c|c|}
\hline & 19.06 .08 & 26.06 .08 \\
\hline \hline$\eta$ & 0.007 & 0.010 \\
\hline$\rho_{\infty}$ & 0.101 & 0.100 \\
\hline$a$ & 5.001 & 5.000 \\
\hline$b$ & 2.000 & 2.001 \\
\hline$g_{\infty}$ & 2.578 & 2.213 \\
\hline
\end{tabular}

Table 4: LMM parameters for correlation structure and volatility function from calibration to ATM caplets. 


\begin{tabular}{|c|c|c|c|c|c|}
\hline Libor $i$ & $\rho_{i}$ & $\kappa_{i}$ & $c_{i}$ & $\sigma_{i}$ & $\begin{array}{l}\text { rel. price } \\
\text { err. }(\%)\end{array}$ \\
\hline 40 & -0.8 & 1.697 & 1.444 & 3.133 & 5.5 \\
\hline 39 & -0.8 & 1.671 & 1.444 & 2.908 & 5.8 \\
\hline 38 & -0.8 & 1.691 & 1.444 & 2.861 & 5.7 \\
\hline 37 & -0.8 & 1.691 & 1.444 & 2.82 & 6.1 \\
\hline 36 & -0.8 & 1.691 & 1.444 & 2.774 & 5.4 \\
\hline 35 & -0.8 & 1.635 & 1.444 & 2.56 & 5.8 \\
\hline 34 & -0.8 & 1.683 & 1.444 & 2.495 & 5.8 \\
\hline 33 & -0.8 & 1.697 & 1.444 & 2.456 & 5.4 \\
\hline 32 & -0.8 & 1.697 & 1.444 & 2.415 & 6 \\
\hline 31 & -0.8 & 1.697 & 1.444 & 2.378 & 5.3 \\
\hline 30 & -0.8 & 1.697 & 1.444 & 2.193 & 5.6 \\
\hline 29 & -0.8 & 1.681 & 1.444 & 2.15 & 5.4 \\
\hline 28 & -0.8 & 1.694 & 1.444 & 2.245 & 5.4 \\
\hline 27 & -0.8 & 1.694 & 1.444 & 2.185 & 5.3 \\
\hline 26 & -0.8 & 1.694 & 1.444 & 2.118 & 5.3 \\
\hline 25 & -0.8 & 1.694 & 1.444 & 2.056 & 5 \\
\hline 24 & -0.8 & 1.694 & 1.444 & 1.994 & 5 \\
\hline 23 & -0.8 & 1.694 & 1.444 & 1.908 & 5.1 \\
\hline 22 & -0.8 & 1.655 & 1.444 & 1.828 & 4.8 \\
\hline 21 & -0.8 & 1.668 & 1.444 & 1.648 & 5 \\
\hline 20 & -0.8 & 1.691 & 1.444 & 1.572 & 5.2 \\
\hline 19 & -0.8 & 1.691 & 1.444 & 1.567 & 4.7 \\
\hline 18 & -0.8 & 1.656 & 1.444 & 1.477 & 4.9 \\
\hline 17 & -0.8 & 1.691 & 1.444 & 1.398 & 5.1 \\
\hline 16 & -0.8 & 1.43 & 1.444 & 1.375 & 5.1 \\
\hline 15 & -0.8 & 1.699 & 1.444 & 1.297 & 5.2 \\
\hline 14 & -0.8 & 1.677 & 1.444 & 1.22 & 4.8 \\
\hline 13 & -0.8 & 1.511 & 1.444 & 1.202 & 6.3 \\
\hline 12 & -0.8 & 1.656 & 1.444 & 1.125 & 6.2 \\
\hline 11 & -0.8 & 1.648 & 1.444 & 1.091 & 6.6 \\
\hline 10 & -0.8 & 1.593 & 1.444 & 1.014 & 7 \\
\hline 9 & -0.8 & 1.696 & 1.444 & 0.937 & 7.1 \\
\hline 8 & -0.8 & 1.301 & 1.444 & 0.923 & 8.8 \\
\hline 7 & -0.8 & 1.576 & 1.444 & 0.91 & 5.5 \\
\hline 6 & -0.8 & 2.245 & 5.87 & 0.956 & 6 \\
\hline 5 & -0.8 & 2.905 & 5.87 & 0.869 & 12.4 \\
\hline
\end{tabular}

Table 5: Stoch. Vol. Libor model calibration to the cap-strike matrix, $r \equiv 0.9$, date 19.06.08. 


\begin{tabular}{|c|c|c|c|c|c|}
\hline Libor $i$ & $\rho_{i}$ & $\kappa_{i}$ & $c_{i}$ & $\sigma_{i}$ & $\begin{array}{l}\text { rel. price } \\
\text { err. }(\%)\end{array}$ \\
\hline 40 & -0.8 & 2.002 & 2.008 & 2.029 & 5.5 \\
\hline 39 & -0.8 & 1.971 & 2.008 & 2.001 & 5.4 \\
\hline 38 & -0.8 & 1.93 & 2.008 & 1.86 & 5.5 \\
\hline 37 & -0.8 & 1.999 & 2.008 & 2.032 & 5.3 \\
\hline 36 & -0.8 & 1.999 & 2.008 & 1.91 & 5.2 \\
\hline 35 & -0.8 & 1.999 & 2.008 & 1.881 & 4.9 \\
\hline 34 & -0.8 & 1.962 & 2.008 & 1.82 & 5 \\
\hline 33 & -0.8 & 1.943 & 2.008 & 1.8 & 4.6 \\
\hline 32 & -0.8 & 1.964 & 2.008 & 1.71 & 4.9 \\
\hline 31 & -0.8 & 1.951 & 2.008 & 1.668 & 4.6 \\
\hline 30 & -0.8 & 1.997 & 2.008 & 1.594 & 4.6 \\
\hline 29 & -0.8 & 1.981 & 2.008 & 1.558 & 4.5 \\
\hline 28 & -0.8 & 1.906 & 2.008 & 1.53 & 4.3 \\
\hline 27 & -0.8 & 1.874 & 2.008 & 1.487 & 4.2 \\
\hline 26 & -0.8 & 2.004 & 2.008 & 1.434 & 4.1 \\
\hline 25 & -0.8 & 1.991 & 2.008 & 1.394 & 4 \\
\hline 24 & -0.8 & 1.935 & 2.008 & 1.36 & 4 \\
\hline 23 & -0.8 & 2.004 & 2.008 & 1.325 & 3.8 \\
\hline 22 & -0.8 & 2.004 & 2.008 & 1.262 & 3.8 \\
\hline 21 & -0.8 & 1.878 & 2.008 & 1.233 & 3.4 \\
\hline 20 & -0.8 & 2.004 & 2.008 & 1.203 & 3.5 \\
\hline 19 & -0.8 & 1.983 & 2.008 & 1.145 & 3.8 \\
\hline 18 & -0.8 & 1.997 & 2.008 & 1.087 & 4 \\
\hline 17 & -0.8 & 1.997 & 2.008 & 1.039 & 4.1 \\
\hline 16 & -0.8 & 1.997 & 2.008 & 1.039 & 4.2 \\
\hline 15 & -0.8 & 1.915 & 2.008 & 0.949 & 3.9 \\
\hline 14 & -0.8 & 4.002 & 8.042 & 0.949 & 5.4 \\
\hline 13 & -0.8 & 3.873 & 8.042 & 0.899 & 5.9 \\
\hline 12 & -0.8 & 3.826 & 8.042 & 0.897 & 6.7 \\
\hline 11 & -0.8 & 3.695 & 8.042 & 0.839 & 6.3 \\
\hline 10 & -0.8 & 3.3 & 8.042 & 0.732 & 7.9 \\
\hline 9 & -0.8 & 3.549 & 8.042 & 0.737 & 7.5 \\
\hline 8 & -0.8 & 3.549 & 8.042 & 0.68 & 9.8 \\
\hline 7 & -0.8 & 3.952 & 11.125 & 0.753 & 7.9 \\
\hline 6 & -0.8 & 3.704 & 13.065 & 0.737 & 5.2 \\
\hline 5 & -0.8 & 4.914 & 16.902 & 0.705 & 14 \\
\hline
\end{tabular}

Table 6: Stoch. Vol. Libor model calibration to the cap-strike matrix, $r \equiv 0.9$, 26.06.08. 